\title{
LIDERAZGO CAÓRDICO PARA ORGANIZACIONES LÍOUIDAS. INNOVACIÓN ESTRATÉGICA ANTE LA INCERTIDUMBRE PARA LA CONSTRUCCIÓN DE FUTUROS DESEABLES
}

\section{“CHAORDIC" LEADERSHIP FOR LIQUID INSTITUTIONS. STRATEGIC INNOVATION VERSUS UNCERTAINTY FOR THE CONSTRUCTION OF DESIRABLE FUTURES}

Recibido: 4 de agosto de 2018

Evaluado: 25 de octubre de 2018

Aprobado: 16 de diciembre de 2018

\author{
María Fernanda Noboa González * \\ Instituto de Altos Estudios Nacionales, Ecuador \\ Orcid: https://orcid.org/0000-0002-4922-4692
}

Cómo citar este artículo: Noboa González, M. F. (2019). Liderazgo caórdico para organizaciones líquidas. Innovación estratégica ante la incertidumbre para la construcción de futuros deseables. Revista Estrategia Organizacional, 8 (1), pp. 27-42. doi: https://doi. org/10.22490/25392786.3170

* Artículo de investigación. Producto de la investigación en el marco de estudios internacionales sobre liderazgo organizacional.

** PhD en Estudios Internacionales (c) (Estudios Estratégicos y de Seguridad). Facultad Latinoamericana de Ciencias Sociales. Licenciada en Comunicación Social, Facultad de Comunicación Social de la Universidad Central del Ecuador. Magister en Relaciones Internacionales (mención en Gobernabilidad y Desarrollo) del Instituto Superior de Posgrado en Ciencias Internacionales. Actual Decana del Centro de Prospectiva Estratégica del Instituto de Altos Estudios Nacionales de Ecuador. Líneas de investigación: metodologías transdisciplinarias; investigación en pensamiento complejo, estratégico y prospectivo. Expertise en inteligencia estratégica y estudios del discurso. Correo electrónico: maria.noboa@iaen.edu.ec 


\section{RESUMEN}

La reflexión teórico-metodológica y profesional en torno a los distintos roles y naturaleza de los liderazgos organizacionales ha cobrado inusitada vigencia en la transición del mundo sólido a líquido. Dicha mutación está obligando a los líderes organizacionales a desafiar su mirada frente a las nuevas cartografías de procesos y sujetos entramados en condiciones espacio-temporales específicas, a partir de la perspectiva unidireccional de orden/desorden. Por ello, se debe repensar la gestión estratégica organizacional. El objetivo de este artículo es desarrollar una investigación teórica-crítica, que desde un enfoque cualitativo, basado en la revisión interdisciplinaria de literatura y varias aproximaciones empíricas, propone un nuevo locus epistémico y analítico, que apunta a pensar de otra manera los liderazgos organizacionales - reflexividad-, sus nuevos desafíos, el deber ético y responsabilidad social, para la construcción de distintos escenarios, comprometidos con el desarrollo integral de nuestra región. Los hallazgos muestran un tímido florecimiento de líneas analíticas de este tipo de liderazgo y el requerimiento de un mayor esfuerzo académico e investigativo para salir de corsé del paradigma lineal. Se concluye en la necesidad de navegar, sin desconcierto, en medio de condiciones de orden/desorden; libertad /control; proacción/reacción; conflictividad/consenso, como estrategia innovativa para pensar y construir conocimiento de alto valor, en apoyo a la toma de decisiones para la gestión organizacional.

Palabras clave: gestión, organización, estrategias caórdicas, prospectiva crítica, mundo líquido, innovación.

\section{ABSTRACT}

The theoretical-methodological and professional reflection surrounding the different roles and natures of organisational leadership has taken on an unusual validity in the transition from a solid to a liquid world. The mutation is forcing organisational leaders to challenge their outlook when faced with the new cartographies of processes and subjects framed in specific spatio-temporal conditions, starting from the unidirectional perspective of order / disorder. Therefore, the strategic organisational management must be rethought. The objective of this article is to develop a theoretical-critical research from a qualitative approach, based on the interdisciplinary review of literature and several empirical approaches. It proposes a new epistemic and analytical locus, which aims to provide alternative ways of thinking about organisational leadership - reflexivity-, its new challenges, ethical duty and social responsibility, for the construction of different scenarios, committed to the integral development of our region. The findings show a timid flowering of 
analytical lines of this type of leadership and the requirement for a greater academic effort to get out of the restrictive linear paradigm. It concludes with the need to concisely navigate, in the midst of order / disorder conditions; freedom / control; proactivity / reaction; conflict / consensus, as an innovative strategy to think and build high value knowledge, in support of decision making for organisational management.

Keywords: Organisation, Management, Chaordic Strategies, Critical Foresight, Liquid World, Innovation.

\section{INTRODUCCIÓN}

La discusión respecto del rol y la naturaleza de los liderazgos para las organizaciones del siglo XXI ha cobrado un especial interés en la región latinoamericana, no solo por los nuevos entramados y dinámicas de los entornos -sociales, naturales y tecnológicos- en los que se inscriben las instituciones, sino por los desafíos que implican para sus actores estratégicos moverse, con ventaja, en las dinámicas altamente competitivas, cambiantes, azarosas que plantea un mundo global, turbulento y líquido, caracterizado principalmente por la fluidez, la creciente vulnerabilidad e incertidumbre (Bauman, 2010).

Los puntos de posición y posicionamiento esperados ${ }^{1}$ por los niveles decisionales para

1 Hago referencia a la capacidad de moverse y evolucionar estratégicamente, desde un punto de partida teniendo un punto de llegada que no es rígido y puede mutar de manera una gestión organizacional eficiente y eficaz han dado un giro insospechado, obligando a los actores clave a la adopción de posturas adaptables y flexibles no solo en sus prácticas, sino partiendo de sus propias percepciones a partir de las cuales ellos han ido construyendo, en el transcurso del tiempo, su sentido de realidad organizacional (Manucci, 2006). Lugar donde todo lo sólido, como sostiene Marshall Berman se va desvaneciendo en el aire, se torna efímero, pues " [...] ser modernos [...] amenaza con destruir todo lo que tenemos, todo lo que sabemos y todo lo que somos" (Bermann, 1988). En cierto sentido, esto es real, porque todas las coordenadas para pensar, vivir y sentir el mundo han cambiado para todos. Es más, las condiciones de orden y desorden que -desde una mirada lineal- eran vistas como contradictorias, en el presente pasan a ser parte de una misma dinámica de coexistencia, aparentemente contradictoria, denominada caórdica (Hock, 2001). Indiscutiblemente, esta 
nueva lógica abre múltiples posibilidades de maniobra para la gestión de un nuevo tipo de liderazgo, que se moverá dentro de sistemas de auto organización adaptación, no linealidad y complejidad, es decir, en medio de constante cambio y evolución. Consecuentemente, los liderazgos se convierten en procesos de autoaprendizaje permanentes, proyectados al aprendizaje de la organización en su conjunto y el logro de ventajas competitivas dinámicas (Unger y Varela, 2017), terreno fértil para la construcción de los mejores escenarios.

En este orden, los líderes de las organizaciones caórdicas obligadamente se enfrentan al desafío de buscar estrategias de cooperación y la responsabilidad de desarrollar permanentes rutas de acción innovadoras para lidiar con los complejos ambientes tecnológicos, políticos, sociales, culturales en los que se insertan las organizaciones (Capriotti, 2013). Para construir así los caminos hacia los futuros deseables, que son múltiples, independientemente que ello implique una planeación por escenarios, previsión o prospectiva estratégica. Lo dicho hasta aquí tiene que ver con un re-pensar la visión, misión y objetivos estratégicos organizacionales, direccionándolos a la construcción de vínculos con los diversos sujetos emparentados con la organización -internos, externos, stakeholders, entre otros-. En este punto, es vital discriminar que los vínculos implican relaciones de confianza, de larga data que los líderes logran construir, mantener y ampliar y que están asociados a la gestión de comportamientos y decisiones de los diversos sujetos $^{2}$ que configuran las organizaciones en su conjunto. Estos, indiscutiblemente, marcan el "estilo organizacional" que a su vez configurará la dupla narración-discurso ${ }^{3}$ organizacional de manera coherente, una herramienta esencial para garantizar la sostenibilidad organizacional, diseño de buenas prácticas de innovación y competitividad para el logro de ventajas competitivas dinámicas. Con ello, indudablemente, se asegura una proyección estratégica pertinente para el mejoramiento continuo, avance y logro de los objetivos organizacionales ${ }^{4}$ (Manucci 2004; 2010). Adicionalmente, a partir de ello, el líder caórdico va generando capacidades de gestión más complejas, abiertas y flexibles, utilizando competencias, habilidades y destrezas que marcan huellas profundas para

2 Aludo a la visión moriniana del sujeto que sin duda es controvertida y paradojal, pero compleja, es una cualidad fundamental del ser vivo, con carácter multidimensional, dejando superadas las dicotomías de las relación de sujeto respecto del objeto

3 Para este trabajo se ha tomado la noción de narración como aquellos significados compartidos y gestionados por los sujetos organizacionales internos, y el discurso como la proyección de esta narración comprometidos en la relación con los distintos entornos en los que se inscribe la organización.

4 Es decir que la organización va asimilando un know how como elemento agregador de valor 
sus futuros desempeños en situaciones parecidas o disímiles, conjugando poder de mando, desempeño laboral, idoneidad para la gestión, especialmente la resolución de problemas con actuaciones integrales y razonadas frente a la incertidumbre (Tobón 2010, p. 91)

Es impensable, entonces, que los líderes ante este tipo de organizaciones continúen pensando en mecánicas de supervisión y control en las lógicas organizacionales; están obligados a desarrollar un pensamiento abierto $^{5}$ al cambio - que también incidirá en su sentir-. En definitiva, sin dejarse perturbar por factores de ruido, disonancias y/o rupturas que vayan emergiendo y configurando situaciones no previstas durante la toma de decisiones, la definición de rutas de acción, en los mismos marcos de intervención en los cuales se mueven las organizaciones, y que responden a coordenadas propias y a condiciones específicas que se desarrollan en nuestras regiones. De modo simultáneo, tiene que ver con humanizar la interacción con el otro, porque al fin y al cabo, los procesos de convivencia y trabajo colaborativo responderán a las diversas capacidades de adaptabilidad sistémica en medio de procesos de intercambio y relacionamiento en la asunción de normas, leyes y pautas construidas colectivamente.

5 Aludo igualmente a superar el pensamiento simplista y reduccionista
De lo que se trata, en definitiva, es pensar y vigilar el presente, debatir el futuro y modelar el/los futuros deseables, de manera tal que, con el diseño de rutas anticipativas, configurar este futuro esperado. $Y$ este implica tener como epicentro el debate del rol y naturaleza del liderazgo para organizaciones líquidas, que paralelamente exige el despertar de una sensibilidad cognitiva y social para lograr incidir en los diversos flujos de interacciones que se generen dentro de la institución, como se ha insistido, sin soslayar la ética en la toma de decisiones, buscando siempre el respeto a la dignidad de los otros y lo otro. ${ }^{6}$

Para matizar lo argumentado, también es preciso mencionar que de la revisión y análisis de la literatura en la región, los nuevos líderes, adaptados sistémicamente -a las organizaciones caórdicas- desarrollan al interior de sus organizaciones nuevos tipos de prácticas congruentes y consistentes que les permiten moverse estratégicamente dentro arenas movedizas (Manucci 2010) -muchas veces desconocidas- que inciden en nuevas lógicas para la toma de decisiones.

De hecho, considerando que todo contexto es un tejido de relaciones que cobra significado para las personas, que a su vez resultan tejidas y sujetadas por el entorno de significación que

6 Colaboradores, medioambiente y entornos 
han sido construidos de esta forma (Tobón, 2010, p. 100), cabe resaltar la relevancia de situarlo y comprenderlo. En suma, en ambientes difusos, cambiantes, inestables y de riesgo permanente la posición de los líderes funciona mediante un sentido de flujos y movilidad que adaptan en sus prácticas cotidianas, trayectorias amplias y mutables, que les permite lidiar con amplia ventaja estratégica frente a situaciones disruptivas, tensiones, agujeros negros, nudos críticos. ${ }^{7}$ En otras palabras los nuevos líderes deberán tener en mente la necesidad de no descuidar la coyuntura en el momento de atender a la perspectiva de largo plazo, orientada a la consolidación de competitividad sustentable y saludable, punto de partida esencial para desarrollar cualquier ejercicio prospectivo ( Baena 2007; Miklos, 2003).

\section{YA NO ES POSIBLE TENER IDEAS SÓLIDAS FRENTE A MUNDO LÍOUIDO. LA APUESTA TEÓRICO-METODOLÓGICA}

De lo argumentado precedentemente, se infiere que el mundo líquido impide conservar el pensamiento sólido asociado a un mundo mecánico. Por tanto, y como ya se ha insistido, el objetivo de largo plazo del líder caórdico es repensar las categorías y conceptos de la gestión

$7 \quad$ Para este trabajo se entienden los agujeros negros y puntos críticos aquellos momentos en que parece todo oscurecerse, no encontrarse salida, no ver opciones para decidir (García, 2006). organizacional, inscrita en el paradigma de la complejidad todo lo cual incide en nuevas formas de abordar la realidad y consecuentemente en la construcción de rutas analíticas específicas, como apoyo a la toma de decisiones. Por ende, este artículo se encamina a una discusión teóricocrítica interdisciplinaria, de los presupuestos del liderazgo caórdico, asumiendo los principios de la complejidad a fin de dotar de algunas pistas de las posibles implicaciones de este redimensionamiento epistémico y pragmático.

En definitiva, la flexibilidad y la capacidad de adaptación sistémica son ineludibles herramientas para que los líderes organizacionales puedan navegar con sensatez en medio de la incertidumbre de un mundo líquido, y -a pesar de ello-, continuar siendo humanos, en un mundo caótico que casi los constriñe a moverse situacionalmente en medio de reglas abiertas, de un mundo turbulento, en medio de la actual crisis de la sociedad, las organizaciones y del sentido mismo de la vida (Elizalde, 2009), sin resquebrajarse, sino por el contrario, asumiendo lecciones aprendidas, incorporándolos a sus saberes significativos de vida.

A propósito de lo dicho hasta aquí, los estudios prospectivos, sobre todo aquellos en- 
marcados en la denominada cuarta generación, ${ }^{8}$ situados principalmente en América del Sur, se centran en el cambio y en la voluntad política y ética de incidencia sobre el presente, modelando el futuro, que es precisamente la naturaleza y el rol del líder caórdico. Su gestión está enmarcada en medio de las totalidades complejas multisectoriales- organizaciones del sector público y privado- en las cuales la presencia de un conjunto de factores, difusos, efímeros, contradictorios, no hacen sino confrontar al hombre con un nuevo espíritu, en donde las instituciones ya no son despersonalizadas, estériles, ni objetivas, sino que se tornan en organismos vivos, con condiciones de autopoiesis en palabras de Maturana y Varela (2003). Esto, igualmente, implica un despertar de su una sensibilidad cognitiva, a partir de la cual los líderes están forzados a mutar de referentes en la modalidad de vasos comunicantes capaces de inspirar a otros, tanto en el afianzamiento y expansión de redes de confianza, como en su capacidad de manejar organizaciones abiertas, multidimensionales y multidireccionales. Es más, esto implica igualmente el diseño de una perspectiva estratégica clara, en medio de parámetros de innovación organizacional en la estructura, procesos y me-

8 Surge desde el 90, primera década del siglo XXI, caracterizada por la incidencia social, la búsqueda de alternativas y de cambio una de cuyas características se fundamenta en el desarrollo y procesos de aprendizaje y respuesta al cambio, es decir el aprendizaje "inteligente" canismos de relacionamiento entre sus actores en la dinámica cooperativa-equitatativa.

Todo esto condiciona, igualmente un claro redimensionamiento y flexibilización de las diversas estrategias encaminadas a la toma de decisiones, que obviamente puede variar, toda vez que en el ambiente volátil actual, nada está esculpideo en piedra; es más dichas estrategias han de ser pensadas como una construcción colectiva. En otras palabras, se refiere a un proceso de "gestión de decisiones", no imposición de ellas. De hecho, las organizaciones líquidas son aquellas con mayor posibilidad de supervivencia, a partir del cambio de la lógica decisional mediante procesos de "desorganización-reorganización" frente a contextos que exigen cooperación y acceso a todos los recursos de manera simultánea para el logro de trabajos más eficientes, con métodos de trabajo más libres, y necesariamente capital humano más preparado.

Es más, en estos nuevos ambients se pone a prueba la capacidad metacognitiva de los líderes actuales (Morin, 1994) que rebasa los saberes fragmentarios y simplistas de la mirada mecánica de la gestión organizacional, para que todo conocimiento o know how institucional se convierta en valor agregado al desarrollo organizacional; de hecho, este conocimiento producto de la experiencia cotidiana de los sujetos en un 
ambiente azaroso, cambiante, inestable, deja en el día a día lecciones aprendidas que permiten a las organizaciones ir adaptándose sistémicamente a condiciones de diversa índole, propias y externas. Es más, va configurando nuevos puntos de abordaje a situaciones sensibles, sin perder de vista la responsabilidad social y el compromiso ético que son indispensables en un liderazgo maduro capaz de sobrellevar exitosamente las nuevas cartografías organizacionales propuestas por un mundo global turbulento, asimétrico y mutante, que interpela a nuestra regiones, porque, en definitiva "[...] el trabajo colectivo y reflexivo desde diferentes regiones [...] por la emergencia de un paradigma de la complejidad puede contener las semillas de una reforma política de matriz humano-céntrica, capaz de repensar la humanidad a escala planetaria (Zoya, 2008, p. 18).

\section{LOS NUEVOS LÍDERES CAÓRDICOS: SUJETOS EMERGENTES A LA ORDEN. EN TORNO A LOS OBJETIVOS}

De todo lo dicho anteriormente, el sentido de liderazgo ha cambiado de rumbo, habida cuenta de que las organizaciones caórdicas no son sólidas sino líquidas, y por ende altamente cambiantes y vulnerables a las dinámicas internas y externas de las organizaciones. En este nuevo ambiente, las aristas de lo comunicacional de dirección -liderazgo-, de marketing
- productos y servicios- y de lo organizacional -cultura-, propiamente dichos, aparecen entramadas, con márgenes borrosos entre ellas, y como es obvio, necesariamente se requieren nuevos tipos de liderazgo en torno a estos inusitados desafíos.

La primera consideración necesaria es que el líder "se constituye en un sujeto" en el sentido moriniano del término, es decir un sujeto multidimensional, vivo, que no es reducible a sus cualidades externas, ni a características psicológicas ni morfológicas, y que por ende cambia la identidad frente a sí mismo -autorreflexividad-. En definitiva, alude a la capacidad de reconocerse frente al mundo (Campero, 2017) $y$, por ende, superar su escisión frente al objeto; en suma, un sujeto como unidad compleja. Desde esta consideración, entonces, la construcción del liderazgo se torna un ejercicio de re-indagarse proyectándose hacia ópticas lógicas relacionales en la organización, a partir del cual él mismo evoluciona, se transforma y crece.

Consideramos que el ejercicio de indagar-se a sí mismo, interpela al sujeto, como a su propio yo, lo que más nos subyuga de esta cuestión es que la pregunta por el yo, implica necesariamente para el sujeto, un saberse siendo él mismo en el mundo y en la relación. (Campero, 2017, p. 136) 
Lo dicho, entonces, condiciona el rol y la naturaleza del liderazgo caórdico, en términos de complejidad organizada. Este hecho no es menor a la hora de pensar la gestión del futuro; los líderes "fluidos" y en constante aprendizaje, necesariamente se inscriben en una nueva manera de pensar el tiempo, superando la perspectiva instrumental, que lo divide en pasado-presente y futuro; se anclan dinámicamente a visiones retrospectivas, transtemporales, circulares y en espiral (Baena, 2004). Esta perspectiva va afianzando al líder en medio de crecimiento discontinuo y variable de las organizaciones, cambios en los niveles de conectividad, indeterminación, disipación ${ }^{9}$ y emergencia. Esto da un giro radical a las concepciones tradicionales de liderazgo que obliga: de un lado, a racionalizar la gestión de manera oportuna, y de otro el logro de vínculos efectivos - para el largo plazo y confianza- con clientes y stakeholders. Esta dinámica está en relación directa con la "vivencia de la otredad", es decir con la capacidad estratégica de moverse en un espacio en donde se evidencian las posiciones y puntos de posicionamiento de los diversos actores que actúan en red, en cuya dinámica el líder debería incidir en un "efecto mariposa" para lograr sinergias y logro de objetivos en los diversos niveles de las organizaciones.

9 Se alude al Segundo Principio de la Termodinámica, a partir del cual las estructuras disipativas, son estructuras coherentes y autoorganizadas en sistemas alejadas del equilibrio, solo aparecen en sistemas abiertos
El líder caórdico está decidiendo dentro de nuevas arenas movedizas desde el entendido de que "los viejos patrones han cambiado , ahora el éxito se basa en la administración por valores" (Blanchard y O'Connors, 1997) y son esos valores los que se convierten en la brújula para la gestión organizacional que proyecta modelos organizacionales y lenguajes para hacer frente a la inestabilidades y lograr la lógica del equilibrio-momentáneo- es decir utilizar sus competencias y habilidades de moverse entre el orden y el caos, flexibilizando la gestión y ampliando la capacidad de configurar imaginarios específicos que permitan la consolidación de escenarios múltiples para la toma de decisiones desde el más alto nivel y buscar respuestas coherentes.

Es más, "en las organizaciones emergentes la autoridad es merecida cuando las personas demuestran su conocimiento y capacidades, y en ningún caso emana del poder de un cargo [...]" (Andrade y Pereira, 2006, p. 13). Esto implica que la verticalidad y horizontalidad en la estructura organizacional van siendo suplantadas por la ubicuidad relacional de personas, a partir de lo cual la autorresponsabilidad, el autogerenciamiento y autodirección van tomando cuerpo, en organizaciones con lógicas de ser y sentirse maduras, en las que el ser, conocer, hacer y convivir en una dinámica de continuum está en permanente mutación (Andrade y 
Pereira, 2006), lo que exige que el sentido del liderazgo vaya consolidando narraciones corporativas que sirvan de guía para las rutas de acción que permitan el modelamiento de los futuros deseados, en los que se pone en juego el sentido futuro de la vida que se espera para la organización y el compromiso ético con los sujetos de ella.

El líder en este tipo de organizaciones, en palabras de Maturana (1996) y de Manucci (2005) tiene la capacidad de deconstruir las creencias cotidianas y cuestionarlas, a fin de comprender los límites que ellas imponen para el diseño del contexto de las organizaciones y sus posteriores dinámicas de actuación en territorios de intervención específicos. Esta capacidad se orienta a situar, de modo pertinente, el espacio organizacional en tanto una construcción colectiva, en medio del cual, el líder se proyecta a la consolidación de ámbitos compartidos que garanticen, asimismo, desarrollar vínculos con el resto de sectores de la sociedad. En definitiva, el desarrollo de nuevas capacidades y competencias facilitarán enfrentarse a los desafíos impuestos por diversos factores externos a la organización y que marcan el compás co-evolutivo de aprendizaje en espiral de las estructuras organizacionales internas, con lo que se garantiza un ir y venir de adentro hacia afuera y viceversa.
Este aprendizaje es la semilla estratégica para el diseño de la gestión y procesos de liderazgo que garanticen solidez interna, vínculos de largo plazo y multiplicación de interconexiones hacia afuera, trascendiendo lo contingente y pasajero. (Manucci, 2005, p.2). En otras palabras el liderazgo caórdico no hace sino garantizar la coexistencia de lo individual -líder- con lo universal -el juego de sujetos diversos-, de manera dinámica, para construir diferentes sentidos significativos y no rígidos para la organización, que puedan ser redefinidos en el tiempo y espacio organizacionales, en respuesta a los mejores cursos de acción para su mejor desarrollo y avance (Andrade y Pereira, 2016, p. 16).

\section{LIDERAZGO CAÓRDICO, ORGANIZACIONES INTELIGENTES, FUTUROS DESEADOS. DISCUSIÓN}

Asumiendo lo dicho anteriormente, los líderes caórdicos, entonces son co-constitutivos y constituyentes de las organizaciones inteligentes, y como tales con una significativa responsabilidad para guía en el modelamiento de escenarios como construcción colectiva, como una trama de símbolos, definidos por el intercambio y gestión de subjetividades. Esto, sin duda, incide en los modelos de "organización" y los tipos de intervención sobre la sociedad, que aunque plena de paradojas y conflictos, más que generar temor o resistencia es una 
oportunidad para que los líderes entramados en el conjunto de sujetos organizacionales logren tejidos sociales asentados en convicción y confianza y encaminen a las organizaciones al cumplimiento de sus objetivos maestros, inmersos en una cultura organizacional de contexto alto. ${ }^{10}$

Los procesos de interacción entre la individualidad y la relacionalidad, aun cuando vayan cambiando en función de la coexistencia de ciclos hegemónicos y paralelos -lo formal e informal de las organizaciones- son vitales para la evolución de las organizaciones como vivientes e inteligentes, con capacidad de adaptación a los cambios de los distintos entornos -social, tecnológico y natural- y, por ende, de innovación y crecimiento, asumiendo los factores emergentes y las sorpresas como ventajas a la hora de inyectar mayor sentido de vida y significado a los organizaciones y sus sujetos (Campero, 2017, p.139). Dichas experiencias múltiples irán sedimentado el "ADN" organizacional, es decir, el aprendizaje individual-colectivo-organizacional como experiencias en donde:

10 Hago referencia al contexto alto en las organizaciones en donde se ha asumido la cultura organizacional como construcción colectiva y se han establecido compromisos implícitos éticos y comunes la desarrollo organizacional,
- Los sujetos aprenden y se transforman permanentemente a partir de sus experiencias vitales en la constitución de sí mismos y de la organización.

- Los colectivos, mediante procesos de interacción a partir de los cuales se construyen mapas, y por ende se abre la posibilidad a la corrección inteligente y consensuada de los errores y redireccionamiento organizacional.

- Los organizacionales, a partir de las experiencias de los aprendizajes colectivos situados y en expansión permanente para crear resultados deseados, donde se establecen nuevos e innovativos patrones de pensamiento que incluyen deseos y aspiraciones colectivos de manera libre, pero unidos por un real sentido de alteridad (Van Eijnatten y Putnik, 2004).

Estos niveles de aprendizaje, obviamente, definen de manera sistémica las decisiones presentes como formas de modelar, desde ya, diversas opciones de futuro, haciendo uso de los aprendizajes significativos, habida cuenta de que las nuevas cartografías del mundo exigen de todas las organizaciones sintonizarse en los cambios, desde el entendido de que la permanencia e inmovilidad precede a la muerte. Por consiguiente, la tarea prospectiva se enmarca en jugar con las cartas de lo potencialmente impredecible e imposible, en medio 
de valores tales como: la creatividad, iniciativa, perseverancia, solidaridad y valentía, apostando por esos futuros que los países de la región latinoamericana merecen no solo diseñar sino vivir, lo que implica inexorablemente un ejercicio prospectivo conjunto de las organizaciones y sus líderes.

Dichos futuros de equidad, inclusión, transformación productiva, innovación, investigación, desarrollo, responsabilidad ética y claridad política, compromiso social, educación, entre otros, parten como sostiene Cordeiro (et al., 2013) de una mirada de los métodos de intervención en el espacio organizacional, ajustando los cursos de acción para incidir de manera directa en la lógica tendencial preponderante, desde un liderazgo que, siendo flexible, inspire y que -como sostiene Hock (1999) - se oriente a la búsqueda intensiva del propósito organizacional, derivando principios, el valor de la gente y la consolidación del concepto clave organizacional, y desde allí considerar la estructura adecuada para el ejercicio de sus mejores prácticas.

Para matizar la lógica argumentativa, también es preciso considerar la construcción de organizaciones saludables derivadas de las acciones de un liderazgo referente, a partir del cual se desarrolle fluidez entre los componentes operativos, cognoscitivos y decisores que mutan rápidamente. En tal sentido, el conocimiento decisorio de un líder caórdico se vincula directamente con el conocimiento en profundidad del espacio organizacional en sí, y del conocimiento situado de cómo debe actuarse para lograr los resultados que se pretenden; en este punto es clave saber hacer, saber dirigir, saber relacionarse estratégicamente mediante el desarrollo de competencias, habilidades y destrezas de interacción y relacionalidad a partir de las cuales los líderes contribuyan a que el resto de actores organizacionales se "compren la pasión" de las mejores prácticas organizacionales al servicio del bien común, contribuyendo a gestionar adecuadamente la diversidad, la complejidad y el cambio orientada a armonizar de manera constructiva la paradoja y el conflicto. Así, en medio de ello orientarla toma de decisiones entre múltiples alternativas. Para ello, y siendo un asunto no menor, es que las teorías sirven como apoyo para organizar nuestra visión de mundo, organizar nuestras miradas y formas que se suponen mejores y más abarcadoras de las precedentes. Mirar, comprender e intervenir en la realidad, como hijos de nuestro tiempo, ese y no otro el valor de las teorías (Pérez y Massoni 2009), en definitiva para iluminar el camino.

El líder caórdico desarrolla una verdadera conciencia de que tomar decisiones también requiere bagaje teórico y no solo prácticas frente a sistemas no lineales y disruptivos. Es más, en medio de ellos se pueden atisbar condiciones 
alejadas del equilibrio y que desafían de manera permanente no solo su marco cognitivo, sino emocional, a la par que los del resto de sujetos de las organizaciones, que se mueven en flujos, con movimientos impredecibles y que se proyectan a expandir su capacidad relacional, del logro de sinergias y objetivos comunes, en el marco de condiciones éticas y responsabilidad co-evolutivamente con los vaivenes del entorno organizacional. De hecho:

Cuando se acercan los límites de crecimiento un sistema caórdico empieza a bifucarse y entra en un período de relativa inestabilidad, en el cual el sistema cambia en una forma no lineal -que nosotros llamamos un cambio transformativo-. (Van Eijnatten, 2004, p. 432).

Por este motivo, en la fase caótica del sistema organizacional, el líder no puede marginalizar las denominadas condiciones iniciales del sistema, toda vez que éstas resultan altamente sensibles, como puntos de partida, que modelarán el estilo del liderazgo, dependiendo de la escala de variación de ellas y sus esquemas de relacionamiento, que a su vez incidirán en la dinámica de las percepciones y posiciones (Manucci, 2004) del resto de actores, que servirán de andamio para la construcción de los futuros deseados.

Lo dicho hasta aquí supone que el logro de los escenarios deseables articulados a la conducción específica de un estilo de liderazgo implica trazar objetivos, alcanzar metas y realizar acciones (Mojica, 2005), reconociendo que ya las tareas presentes son los embriones palpables del futuro. Ante ello es una exigencia la ejecución permanente de ejercicios colectivos de construcción y legitimación, dentro de la organización, de una pensamiento prospectivo-caórdico que de manera sistémica, abierta y flexible permita interacciones constructivas, creativas, también mediante una revisión permanente del mapa organizacional, a fin de consolidar procesos de anticipación, orientados de manera pertinente a la prevención de conflictos y al fomento de la cooperación (Baena, 2004, p.121) dentro de la organización y fuera de ella.

Además, desde el entendido de que lidiar con sistemas caórdicos implica, entre otros factores, "un intento meta teórico para acomodar lentes conceptuales" (Edwards, 2013, p. 160) son precisamente estos lentes los que permitirán a los actores estratégicos organizacionales, construir liderazgos que garanticen significados organizacionales compartidos, sinergia de posiciones entre actores organizacionales, flexibilidad y adaptabilidad de respuestas frente a los diversos niveles de cambio - gran escala, escala intermedia y pequeña escala-, multidimensionalidad de respuestas frente a situaciones sensibles o de crisis, y, finalmente, 
creatividad para concretar los futuros soñados, que permitan que la organización vaya escalando de los puntos de posición actuales a los puntos de posicionamiento estratégico esperados. Es decir que el diseño de la acción organizacional es un proceso imaginativo relacional (Bas, 1999) que mediante la reducción de la incertidumbre provocada por la aleatoriedad de los eventos futuros y en base a la gestión del conocimiento organizacional de alto valor, permiten sacar ventaja estratégica en la toma de decisiones clave para la mejor maniobra organizacional y direccionar los futuros posibles, probables y soñados.

Finalmente, se insiste en que los líderes caórdicos se mueven en sistemas abiertos e impredecibles, que a veces revelan autonomía entre sus componentes, a veces independencia, y que produciendo emergencias múltiples con propiedades nuevas, están lejos de poder enmarcar su comprensión, mediante tendencias rígidas, lo que exige la gestión desde nuevas racionalidades capaces de intervenir tomando en consideración que:

[...] si la significación y el valor de cada elemento de una estructura dinámica o sistema están íntimamente relacionado con los demás, si todo es función de todo, y si cada elemento es necesario para definir a los otros, no podrá ser visto "en sí" de forma aislada, sino a través de la posición y de la función o papel que desempeña en la estructura (Martínez, 2011).

En definitiva, el ejercicio del liderazgo caórdico es un reto respecto de la gestión desde nuevos enfoques teórico-metodológicos: una praxis continua sobre actividades creadoras; la disposición efectiva a la construcción de redes, en un constante clima de libertad mental, una atmósfera que estimule y valore el pensamiento divergente, la diversidad y la crítica fundada, a fin de encaminar el proceso de construcción colectiva para el logro de los objetivos clave de la organización.

\section{DE LAS EXPECTATIVAS A LAS CONCLUSIONES}

De toda la reflexión precedente y en alineamiento a la postura onto-epistémica de este artículo, sería pensar de manera reduccionista y simple pretender esbozar conclusiones cerradas y fragmentarias y definitivas, cuando se plantea que el mundo es líquido. Es una nomenclatura aceptada en este nuevo tiempo la inflexión, la disrupción, la volatilidad, la incertidumbre, la inestabilidad, por lo que este trabajo no ha hecho sino mostrar críticamente el ambiente en el que se desarrollan los líderes en pleno siglo XXI encaminados al XXII, para la gestión de los futuros posibles, saliendo del corsé impuesto por el pensamiento mecánico, correspondiente a una visión cartesiana del mundo. 
La monumentalidad del liderazgo organizacional pierde peso. El sentido del liderazgo deja de estar esculpido en piedra, como una receta inamovible y da paso a la legitimación de hombres de acción, visionarios -operadores públicos, privados, organizacionales, entre otros- adaptables y flexibles, capaces de utilizar estratégicamente sus marcos cognoscitivos y operativos para liderar en medio de condiciones de orden-caos. Es decir, que la apuesta del liderazgo caórdico se inscribe en la capacidad de bregar en medio de entornos complejos con constituyentes heterogéneos, en relaciones paradójicas de lo uno y lo múltiple, en medio de dimensiones sistémico-organizacionales, en parámetros de propiedades y comprensiones diversas de los sujetos organizacionales y en respuesta a interrogantes de una realidad polifacéctica y cambiante. El reto está lanzado: los líderes de organizaciones inteligentes tienen la palabra.

\section{REFERENCIAS}

Baena, G. (2004). Prospectiva Política. Guía para su comprensión y práctica. Ciudad de México, México: Universidad Nacional Autónoma de México. Proyecto PAPIME. Prospectiva Política.

Baena, G. (abril de 2007). Técnicas de Prospectiva Social. Workin Paper. Estudios de Futuro N. 5. Ciudad de México, México: Facultad de Ciencias Polticas y Sociales. Universidad Autónoma de México.
Bas, E. (2008). Prospectiva. Cómo usar el pensamiento sobre el futuro. Barcelona, España: Ariel S.A.

Bauman, Z. (2002). Modernidad Líquida . México: Fondo de Cultura Económica.

Bauman, Z. (2010). Vida líquida. Barcelona, España: Paidós Ibérica .

Bermann, M. (1988). Todo lo sólido se desvanece en el aire. La experiencia de la modernidad. Barcelona, España: Siglo XXI, editores s.a. c.v.

Campero, M. B. (s.f.). Un individuo, un sujeto. El yo como una unidad compleja. Revista de Filosofía, 42(1), 135-151. doi: http://dx.doi.org/10.5209/RESF.55453

Capriotti, P. (2013). Planificación estratégica de la imagen corporativa. Málaga, España : Ariel .

Corderio, J. L. et. al. (2013). Latinoamerica 2030. Estados Unidos: The Millenium Project. United Nations University.

Edwards, M. (2013). A Metatheorical Evaluation of Chaordic Systems Thinking. Systemas Research and Behavioral Science, 160-180. doi: 10.1002/sres.2193

Elizalde, A. (2009). Navegar en la incertidumbre. El desafío de seguir siendo humano en un mundo sin certezas . Bogotá, Colombia : Editorial Corporación Universitaria. 
García, R. (2006). Sistemas complejos. Conceptos, métodos y fundamentación epistemológica de la investigación interdisciplinaria. Barcelona, España: Gedisa .

Hock, D. (1999). Birth of the Chaordic Age. San Francisco C.A., USA: Berett-Koehler.

Manucci, M. (2005). Narraciones corporativas, estrategia y futuro de las organizaciones. Razón y Palabras, 43.

Manucci, M. (2006). La estrategia de los cuatro círculos . Barcelona, España : Norma.

Martínez, M. (2011). Paradigmas emergentes y ciencias de la complejidad. Opción(65), 45-80. Recuperado en: https://dialnet.unirioja.es/ejemplar/298446

Maturana, H. (1996). El sentido de lo humano . Santiago de Chile, Chile : Dolmen Ediciones S.A.

Maturana, H \& Varela, F. (2003). Autopoiesis: La Organización de lo vivo. Santiago de Chile, Chile: Editorial Universitaria Lumen.

Miklos, T. (2003). Las decisiones políticas. De la planeación a la acción . México: Instituto Federal Electoral, Siglo XXI Editores.
Mojica, F. J. (2005). La construcción del futuro . Concepto, modelo de prospectiva estratégica, territorial y tecnológica . Bogotá, Colombia : Convenio Andrés Bello. Universidad Externado de Colombia .

Morin, E. (1994). El Método III. El conocimiento del conocimiento . Madrid, España : Cátedra.

Pereira, R. A. (206). Las fárbices caórdicas. Nuevos lenguajes organizacionales en la era de la complejidad. (CISPO, Ed.) Polis. Revista latinoamericana. Recuperado en: http://polis.revues.org/4974

Putnik, F. M. (2004). Chaos, Complexity Learning and the learning organization. Emerald Insight, 11(6), 418-429. doi: http://dx.doi.org/10.1108/09696470410548782

Varela, K. U. (2017). Complejidad, competitividad e innovación en los estados mexicanos. La urgencia de una política industrial, integral y diferenciada. Documentos de trabajo (605). Ciudad de México, México: Centro de Investigación y docencia económicas.

Zoya, L. R. (2008). Complejidad: La emergencia de nuevos valores epistémicos y no epistémicos en la historia de la ciencias contemporánea . I Encuentro Latinoamericano de la Metodología de las Ciencias Sociales. La Plata . 